\title{
Early Experience with a Hepatobiliary and Pancreatic Quality Improvement Program
}

\author{
Derek O'Reilly, Rachel Edmiston, Pooja Bijoor, Rahul Deshpande, Nicola de'Liguori Carino, Basil Ammori, David J. Sherlock
} North Manchester General Hospital. M8 5RB

\begin{abstract}
This project aims to assess the impact of the introduction of a hepatobiliary and pancreatic (HPB) Quality Improvement Program (QIP) on postoperative complications following liver, biliary and pancreatic surgery.

A prospective analysis of postoperative complications over a six month period was performed. Complications were analysed and graded according to internationally agreed definitions. Justification was sought and errors identified. Weekly meetings were performed to review each complication enabling an action plan to be created to prevent future recurrence. Rates were compared with previously audited and published results, using the chi-square test. A total of 326 procedures were performed over the six months including 30 pancreatectomies, 45 liver resections and 251 other procedures. 37 patients developed complications (11.3\%) with 47 complications in total including two deaths. Using the ISGPS grading, eight complications were identified; two grade A, four grade B and two grade $C$. There were three grade A ISGLS complications and one grade B. 30 complications were justified as unavoidable, 16 as avoidable and one as indeterminate. Action plans included continued monitoring $(n=41)$, formulation of new policy $(n=3)$, individual counselling $(n=4)$, educational offering $(n=4)$. When compared with 2010 complication rates, 114 complications occurred in 233 liver operations during the baseline period, compared with 17 complications in 45 liver operations during the QIP period, OR=0.63 (95\% Cl: 0.33 to 1.22), $p=0.17$ and 86 complications occurred in 99 pancreatic resections during the baseline period, compared with 20 complications in 30 pancreatic resections during the QIP period OR=0.30 (95\% Cl: 0.12 to 0.79$), p=0.01$
\end{abstract}

The HPB QIP is a rigorous approach to grade every complication and death. A statistically significant reduction in pancreas related complications has already been obtained. Further work is required to determine the persistence and magnitude of this quality improvement.

\section{Problem}

Surgical morbidity and mortality are significant public health concerns. Preventable post-operative complications result in needless patient suffering, prolonged hospital stay and wasted hospital resources. Complications following hepatobiliary and pancreatic (HPB) surgery are among the highest of any surgical speciality, due to a combination of patient and procedure-related factors (1).

Morbidity and mortality rates in our department have been well documented, as part of regular audit cycles. In 2010, we published an analysis of 99 consecutive patients undergoing pancreatic resection between January 2006 and September 2008 (2). In total, there were 86 post-operative complications occurring in 55 patients (55.6\%). Our in-hospital or 30-day mortality rate was 3\%. For liver surgery, contemporaneous audit results for the two-year period 2009-2010 showed that there were 114 complications following 233 consecutive liver resections and a mortality rate of $1.3 \%$

We have developed a specific HPB Quality Improvement Program (QIP) and introduced this in September 2012, with the purpose of reducing post-operative morbidity and mortality rates.

\section{Background}

Despite the effects of centralisation for complex surgery, advances in pre-operative patient optimisation, operative techniques and postoperative care, liver and pancreatic surgery remain associated with a high morbidity and a significant mortality.

A major systematic review of liver resection for colorectal metastases has assessed the published evidence for its efficacy and safety (1). 30 independent studies met all the eligibility criteria for the review and data on 30-day mortality and morbidity only were included from a further nine studies. Death within 30 days of hepatic resection was reported by 24 studies, ranging from $0 \%$ to $6.6 \%$ of patients (median $2.8 \%$ ). The commonest causes of postoperative morbidity were: wound infection (5.4\%), generalised sepsis $(4.6 \%)$, pleural effusion $(4.3 \%)$, bile leak $(4.0 \%)$, perihepatic abscess (3.0\%), hepatic failure $(2.8 \%)$, arrhythmia $(2.8 \%)$, postoperative haemorrhage $(2.7 \%)$, cardiac failure $(2.4 \%)$ and pneumonia (1.9\%). Similarly, a major review of contemporary standards in pancreatic surgery found a mortality rate in high-volume centres of 1 to $4 \%$ (3). Intra-abdominal abscess following pancreatic resection occurred in 1 to $12 \%$ of patients, postoperative haemorrhage in 2 to $15 \%$, pancreatic fistula ranged from 2 to $24 \%$ (depending on definition), and the incidence of delayed gastric emptying ranged from 14 to 70 $\%$.

In general, the most significant efforts to decrease surgical morbidity and mortality have included the World Health 
Organisation (WHO) Surgical Safety Checklist and the National Surgical Quality Improvement Programme (NSQIP) of the American College of Surgeons (ACS). The WHO Surgical Safety Checklist is designed to improve team communication and consistency of care and hence decrease complications and deaths as a result of surgery. A study of the implementation of the WHO checklist in eight hospitals across the world showed a reduction in the complication rate of $36 \%$ and inpatient death by $47 \%$ (4). As a consequence, a Patient Safety Alert was issued in the UK in January 2009 by the National Patient Safety Agency, requiring NHS organisations to ensure the $\mathrm{WHO}$ checklist is completed in every patient undergoing a surgical procedure. The NSQIP provides a prospective, peer-controlled, validated database of pre-operative to 30 days post-operative surgical outcomes based on clinical data. In 2009, a study evaluating 118 hospitals using the ACS NSQIP from 2005-2007 found that every year these hospitals were able to prevent 250-500 complications, save 12-36 lives and reduced costs by millions of dollars (5). In the UK, the implementation of a systematic trauma quality improvement system was associated with a fall in the mortality rate among patients presenting in shock (6).

\section{Baseline Measurement}

In 2010, we published an analysis of 99 consecutive patients undergoing pancreatic resection between January 2006 and September 2008 (2). In total, there were 86 post-operative complications occurring in 55 patients $(55.6 \%)$. The International Study Group for Pancreatic Surgery (ISGPS) have provided definitions for the common pancreas-specific complications following pancreatic surgery, namely: pancreatic fistula (PF) (7), post-pancreatectomy haemorrhage (PPH) (8) and delayed gastric emptying (DGE) (9). Our rates for PF, PPH and DGE were 19\%, $5 \%$ and $21 \%$ respectively. Each complication is graded for severity of impact, with grade $\mathrm{A}$ representing the mildest and grade $\mathrm{C}$ the most severe complication.

See supplementary file Methods: Table 1- Pancreas post-operative morbidity 2010 series (2)

See supplementary file Methods: Figure 1- Pancreas post-operative complications according to the ISGPS definitions (2)

The 30-day or in-hospital mortality was $3 \%(n=3)$. One patient developed severe endocarditis, a second patient developed a pancreatic anastomotic leak and died of respiratory failure, and the third patient aspirated in the immediate post-operative period, despite a nasogastric tube and died of respiratory failure.

Contemporaneous audit results for the two-year period 2009-2010 showed that there were 114 complications following 233 consecutive liver resections and a mortality rate of $1.3 \%$. The International Study Group for Liver Surgery (ISGLS) have provided definitions for the liver-specific complications following liver surgery, namely: bile leak $(\mathrm{BL})(10)$, post-hepatectomy haemorrhage $(\mathrm{PHH})$ (11) and post-hepatectomy liver failure (PHLF) (12). However, these definitions were only published in 2011 and therefore not utilised at the time of the baseline audit. Our rates for $\mathrm{BL}, \mathrm{PHH}$ and PHLF were $6.4 \%, 11.2 \%$ and $13.7 \%$. There was 3 post-operative deaths following liver surgery (1.3\%): 2 from liver failure and 1 from a cerebrovascular accident on day 9 .

See supplementary file: ds2497.docx - "Methods"

\section{Design}

The HPB QIP is a system for recording, analysing and reducing post operative morbidity and mortality. The inspiration for this system was the approach outlined in the Trauma Performance Improvement Reference Manual produced by the Performance Improvement Subcommittee of the American College of Surgeons Committee on Trauma in 2002 (13). Modifications were made to incorporate the many differences and specificities of HPB surgery.

See supplementary file Methods: the HPB QIP Proforma.

The process:

The Weekly QIP Meeting:

A weekly QIP meeting is undertaken. This is consultant led but attended by the whole team, who participate via the presentation and analysis of each complication. For each complication, a proforma has been completed, containing patient demographics, the date of surgery and the type of complication that has developed. This forms the starting point of the QIP discussion.

Categorisation \& Grading:

The type or nature of each complication is categorised. Internationally agreed definitions, such as those produced by the ISGPS (7)(8)(9) and the ISGLS (10)(11)(12) are used where applicable. For general complications, the Clavian-Dindo system is used (14). This also grades according to increasingly significant clinical impact

Justification and Error Analysis:

An assessment is made as to whether each complication was justifiable, unavoidable, or consistent with reasonable and prudent practice given the situation or clinical data available or not justifiable, avoidable and not consistent with the standards of the practice or service. For complications considered not justifiable, a root cause analysis is undertaken to identify the errors that gave rise to the complication. Errors can occur at any time in the patient pathway, from pre-operative patient selection, intra-operative errors to errors made in post-operative management.

Action Plans:

An action plan is created in each case where a complication has been considered not justifiable. Action plans include: on-going monitoring, audit, new policy formation, further departmental training or education as well as individual discussion and counselling. This allowed us to make changes as soon as problems were identified to help achieve a reduction in operative complication rates. 
BMJ Quality Improvement Reports

Mortality Analysis: Each death is analysed in a similar way and a judgement made as to whether the death was preventable or not, using the categories: Not preventable, Possibly Preventable,

Probably Preventable and Preventable.

Return to theatre and readmission rates:

Each of these adverse events is also analysed, errors identified and an action plan formulated.

Sharing Information:

Consolidated data and discussion of each avoidable complication and death is then presented to the wider surgical department at the regular audit meetings. These meetings are multidisciplinary and provide a platform for shared learning.

Statistical analysis:

Categorical data such as complication rates were summarised for all patients using proportions and bar graphs. Numerical data such as length of stay were summarised for both groups (those with complications and those without) using medians and interquartile ranges. Complication rates were compared with previously audited and published results, using the chi-square test. Statistical analyses was performed using StatsDirect statistical software version 2.7.7 and Stata/IC version 10.0 .

\section{Strategy}

The impact of this proposal will be measured in terms of Quality Improvement. The Institute of Medicine report "Crossing the Quality Chasm" (15), defined quality based upon six aims for improvement, which have become accepted definitions of the dimensions of quality: effectiveness, efficiency, patient centeredness, safety, timeliness and equity.

Effectiveness; "avoiding both the overuse of ineffective care and the underuse of effective care".

Measured by: Complication rates (according to definitions of the ISGPS, ISGLS and Clavien system) and Mortality rate

Efficiency; "The reduction of waste....and the total cost of care".

Measured by: Total length of stay and total hospital costs

Patient Centeredness; "respecting the individual patient's choices and needs".

Measured by: Patient satisfaction questionnaire

Safety; "avoiding injuries to patients from the care that is intended to help them".

Measured by: Complication rates and Mortality rate

Timeliness; "continually reducing waiting times and delays".
Equity; "closing racial and ethnic gaps".

Measured by: Patient satisfaction questionnaire and demographic data from patient registration.

By introducing this project to the department we aim to reduce complication rates and in turn improve patient satisfaction, reduce hospital stay, prevent avoidable readmissions and in doing so improve the quality of care delivered.

See supplementary file Methods: an example of a potential cost saving.

\section{Results}

\section{Current Complication rates:}

A total of 326 HPB operations were performed over a six month period. 30 pancreatic resections were performed with 15 patients developing 20 complications (50\% complication rate). 45 Liver resections were performed with 14 patients developing 17 complications ( $31 \%$ complication rate) . 202 cholecystectomies were performed with 6 patients developing a total of 6 complications ( $3 \%$ complication rate). In the remaining 34 miscellaneous procedures, there were 4 further complications in 4 patients. There were 2 deaths; one following pancreatectomy and one after liver resection.

Pancreatic fistula was the most common complication with a rate for all ISGPS grades of $23 \%$. The group "other complications" included two intra abdominal collections and one each of the following: pressure sore, gastric fistula, iatrogenic splenic injury, incisional hernia, Chyle leak, CVA, GI bleed, acute kidney injury, central line infection, enteric fistula and haematoma.

See supplementary file Results for Figure 2: Complications by type and frequency

Grading of complications:

Post pancreatic resection:

In total 7 pancreatic fistulae were identified (23.3\%): 2 grade A, 3 grade $B$ and 2 grade $C$. There was 1 grade $B$ delayed gastric emptying (3.3\%). There were no cases of post pancreatectomy haemorrhage. There were 12 other post-pancreatectomy complications graded according to the Clavien-Dindo system: 3 grade 1,1 grade 2, 0 grade $3 a, 1$ grade $3 b, 3$ grade $4 a, 3$ grade $4 b$ and 1 grade 5 .

Post hepatectomy:

In total 3 bile leaks were identified (6.6\%): 2 grade $A$ and 1 grade $B$. One patient developed grade $A$ liver failure. There were no posthepatectomy haemorrhages. There were 12 other posthepatectomy complications graded according to the Clavien-Dindo system: 5 grade 1, 3 grade 2, 3 grade $3 a, 0$ grade $3 b, 1$ grade $4 a, 0$ grade $4 b, 1$ grade 5 . 
Justification:

Thirty complications were justified as unavoidable and sixteen as avoidable (33.3\%).

See supplementary file Results: Figure 3 Justification by procedure

Errors:

Following a root cause analysis in each case where the complication was considered unjustified or avoidable, 20 different errors were identified in 16 sixteen complications.

See supplementary file Results: Figure 4 Breakdown of errors identified following each procedure for unjustified complications

Action plans:

The majority of action plans included tabulation and tracking $(\mathrm{n}=$ 41). Other actions included: formulation of new policy $(n=2)$, individual counselling $(n=4)$ and educational offering $(n=4)$.

Comparison with baseline audit data from 2009 and 2010:

114 complications occurred in 233 liver operations during the baseline period, compared with 17 complications in 45 liver operations during the QIP period, OR=0.63 ( $95 \% \mathrm{Cl}: 0.33$ to 1.22 ), $\mathrm{p}=0.17$

86 complications occurred in 99 pancreatic resections during the baseline period, compared with 20 complications in 30 pancreatic resections during the QIP period, OR=0.30 ( $95 \% \mathrm{Cl}: 0.12$ to 0.79$)$, $\mathrm{p}=0.01$

Return to theatre and Readmission rates :

5 patients were readmitted during the six month period. 3 following pancreatic resections, 1 following an open cholecystectomy and one following biliary reconstruction. Two of these patients (including the open cholecystectomy and following one pancreatic resection) required a re operation. $A$ further 4 patients required a return to theatre. Of the 6 re operations, 2 were secondary to pancreatic fistula, 2 were secondary to bile leaks and 1 each following the development of post-operative bleeding and a gastrojejunostomy leak.

Deaths:

2 of the 47 complications were deaths, giving a 90 day or in-hospital mortality rate of $0.61 \%$ for all operations. Mortality rates following liver resection and pancreatectomy were $2.2 \%$ and $3.3 \%$ respectively.

On mortality analysis, that following liver resection occurred after a right hemihepatectomy with synchronous right hemicolectomy. Postoperatively the patient developed severe ARDS and died of respiratory failure. In this case, errors identified included patient selection and intravenous fluid prescribing. This death was graded as probably preventable. The action plan implemented in this case involved formulation of new policy, individual counselling and educational offering. The death following pancreatectomy was due to a post-operative myocardial infarction. Despite cardiological assessment and stress testing pre-operatively, the error identified was of patient selection and the death deemed possibly preventable. The action plan was formation of a new policy or procedure.

See supplementary file: ds2496.docx - "Results"

\section{Lessons and Limitations}

Lessons learnt:

This project has demonstrated that a HPB QIP is an effective way to address the significant clinical problem of high morbidity rates following liver and pancreatic surgery. Success requires a commitment to quality improvement from the clinical team, the organisation the team works in and the wider National Health Service (NHS). Only once a culture of safety and harm reduction is considered a paramount objective can real progress be made.

It is therefore essential that a program such as this has the wholehearted supported of hospital management. The clinicians leading the program must have the ability to recommend and introduce change, otherwise the process becomes merely an exercise in data collection. Consequently, an understanding of change management and how to overcome vested interests opposed to change, in a diplomatic but effective manner, is desirable.

Sustainability requires that the current upsurge in interest in Quality Improvement in the NHS is maintained and that lessons are learnt from, for example, the disastrous outcomes and standards of care outlined in the Francis report (16). Corporate self-interest and targetdriven management should never again be allowed to over-ride the pursuit of safe clinical care. Whether this particular HPB QIP can continue to make improvements in the reduction of surgical complications in a sustained, year-on-year fashion remains to be seen but is the focus of our on-going efforts and future work.

Limitations:

Potential limitations of this study include ascertainment bias, underpowering and observer bias.

Complication rates depend not just on the quality of surgical skill and perioperative care, but also on definition and on the rigour with which complications are sought. The highly sensitive definitions provided by the ISGPS and ISGLS are therefore valuable as they allow, for the first time, comparison between institutions in a valid manner. Some (mainly those produced by the ISGLS) were not published until 2011, so complications identified during the baseline period were either retrospectively applied or identified according to contemporary standards. The rigour with which complications were sought was high during both periods (as we published an analysis of our complications during the baseline period) but the precise purpose of a weekly QIP meeting is the completeness of case 
ascertainment. The effects of this limitation, however, is to tend to make improvements more difficult to identify.

The current complication rates were based on an analysis of the first six months after implementation of the HPB QIP. This compares with at least a two-year period of baseline measurement. Differences in complications may not have emerged for liver surgery because of the relatively small numbers during the current period and the study is certainly underpowered to detect a difference in mortality rates between the two periods.

It may be argued that the reductions in the complication rates already achieved were a result of the intense scrutiny a department comes under when a QIP is introduced (a surgical Hawthorne effect (17)) rather than as a result of changes introduced as part of an action plan. Ultimately it matters less whether Quality Improvement is achieved as a result of observation than by changed practice, than that its objectives are achieved and complication rates reduced. Quality Improvement is most likely a complex interaction of both factors.

\section{Conclusion}

In summary, a HPB QIP has been designed and introduced to our unit. Early results show that complications can be significantly reduced with this approach.

The scale of the problem of post operative morbidity and mortality after this type of surgery remains high. These high complication rates reflect not just the relatively elderly patient population with comorbidities, the technically challenging nature of the surgery but also sensitive definitions and thorough case ascertainment.

The HPB QIP is a rigorous approach to grade every complication and death. Errors are identified and an action plan made in each case. A statistically significant reduction in pancreas related complications has already been obtained. Further work is required to achieve and maintain ongoing reductions in complication rates and postoperative deaths.

\section{References}

1. Simmonds PC, Primrose JN , Colquitt JL et al. Surgical resection of hepatic metastases from colorectal cancer: a systematic review of published studies. Br J Cancer 2006 ; $94: 982-99$

2. Knight BC, Kausar A, Manu M et al. Evaluation of surgical outcome scores according to ISGPS definitions in patients undergoing pancreatic resection (2010). Dig Surg; 27:367-374

3. Alexakis N, Halloran C, Raraty M, Ghaneh P, Sutton R, Neoptolemos J. Current standards of surgery for pancreatic cancer. Br J Surg, 2004, 91(11):1410-27

4. Haynes A, Weiser TG, Berry WR, et al A Surgical Safety Checklist to Reduce Morbidity and Mortality in a Global Population. New England Journal of Medicine 2009: 360(5): pp $491-495$
5. Hall BL., et al. "Does Surgical quality improve in the American College of Surgeons National Surgical Quality Improvement Program." Ann Surg. 2009; 250:363-376.

6. O Reilly D, Mahendran K, West A, Shirley P, Walsh M, Tai N. Opportunities for improvement in the management of patients who die from haemorrhage after trauma. BJS 2012, 9096

7. Bassi C, Dervenis C, Butturini G, Fingerhut A, Yeo C, Izbicki J,...Buchler M Postoperative pancreatic fistula: an international study group (ISGPF) definition. Surgery, 2005; 138(1):8-13

8. Wente M, Veit J, Bassi C, Dervenis C, Fingerhut A, Gouma $\mathrm{D}, \ldots$ Buchler W. Postpancreatectomy haemorrhage (PPH): an international study group of pancreatic surgery (ISGPS) definition. Surgery, 2007; 142(1):20-5

9. Wente M, Bassi C, Dervenis C, Fingerhut A, Gouma D, Izbicki J . Delayed gastric emptying (DGE) after pancreatic surgery: a suggested definition by the international study group of pancreatic surgery (ISGPS). Surgery, 2007;142(5):761-8

10. Koch M, Garden J, Padbury R, Rahbari N, Adam R, Capussoi $L, \ldots$ Weitz $\mathrm{J}$. Bile leakage after hepatobiliary and pancreatic surgery: A definition and grading of severity by the international study group of liver surgery. Surgery, 2010;149(5):680-688

11. Rahbari N, Garden J, Padbury R, Maddern G, Koch M, Thomas $\mathrm{H}, \ldots$ Weitz $\mathrm{J}$. Post-Hepatectomy haemorrhage: a definition and grading by the international study group of liver surgery (ISGLS). HPB,2011; 13, 528-535

12. Rahbari N, Garden J, Padbury R, Brooke-Smith M, Crawford M, Adam R,...Weitz J . Posthepatectomy liver failure: A definition and grading by the international study group of liver surgery (ISGLS). Surgery, 2010; 149(5):713-724

13. American College of Surgeons Committee on Trauma (2002) Trauma Performance Improvement reference manual. Retrieved from http://www.facs.org/trauma/publications/traumaon.html

14. Clavien P, Barkun J, de Oliveira M, Vauthey J, Dindo D, Schulick R,...Makuuchi M . The Clavien-Dindo Classification of surgical complications five year experience. Ann Surg, 2009; 250: 187-196

15. Committee on Quality of Health Care in America. 'Crossing the Quality Chasm: A new health system for the 21st century' Institute of Medicine. March 2001

16. The Mid Staffordshire NHS Foundation Trust Inquiry. Independent inquiry into care provided by Mid Staffordshire NHS Foundation Trust: January 2005 - March 2009

17. Birkmeyer J. Progress and challenges in improving surgical outcomes. BJS 2012, 99:1467-1469

\section{Declaration of interests}

Nothing to declare

\section{Acknowledgements}


BMJ Quality Improvement Reports

None 